Editorial

\title{
Radiation Exposure and the Neuroanesthesiologist
}

\author{
Jeffrey J. Pasternak ${ }^{1}$ \\ ${ }^{1}$ Department of Anesthesiology and Perioperative Medicine, Mayo \\ Clinic College of Medicine, Rochester, Minnesota, United States
}

J Neuroanaesthesiol Crit Care:2021;8:1-2

I have done it many times, I have stood there unprotected while I cared for a patient having a quick postoperative computerized tomography (CT) scan of the head. I have walked into an interventional radiology room during a procedure, without wearing a lead apron, to quickly ask the radiologist a question about the next patient. Most would easily agree that I would be putting myself at unnecessary risk from radiation exposure in these two situations. I have also stood there many times, wearing a lead apron and thyroid shield, at the head of the bed, talking to a patient to try to comfort them during a cerebral angiogram. Am I really completely protected from radiation risk in this latter scenario?

The term "radiation" refers to electromagnetic waves or moving subatomic particles. Radiation can be divided into ionizing or nonionizing radiation based on whether or not the electromagnetic waves or particles have the energy to cause electrons to overcome the electrostatic forces that bind them to atomic nuclei. As human beings living on the Earth moving through outer space, we are all constantly exposed to radiation from a variety of sources. For the average human, most radiation exposure comes from the decay of radioactive elements in the earth and environment as well as from cosmic rays from outer space. However, in countries that readily use medical imaging, medical radiation exposure can account for up to half of the annual radiation exposure for the average person in that country. ${ }^{1}$ Radiation exposure from medical imaging may even be responsible for 1 to $3 \%$ of cancers worldwide. ${ }^{2}$

As anesthesiologists who care for patients with neurological diseases, we are most likely to encounter radiation via imaging techniques that utilize X-rays, most commonly in the form of CT imaging and fluoroscopy in interventional radiology suites. X-rays are electromagnetic waves with a wavelength of $\sim 0.01$ to $10 \mathrm{~nm}$ and carry enough energy to easily ionize atomic and molecular targets. In fact, X-ray imaging is, in part, based on the ability of X-rays to interact with substances in their path as differential scattering and ionization of atoms and molecules in different tissues lead to differences in the appearance of these structures on imaging.

Address for correspondence

Jeffrey J. Pasternak, MS, MD,

Mayo Clinic College of Medicine,

200 First Street, SW, Rochester,

MN 55905, United States

(e-mail: pasternak.jeffrey@mayo.edu).
Less common radiation-based techniques encountered in patients with neurological diseases include positron-emission tomography, single photon emission computerized tomography, and gamma knife. These latter techniques either directly, or by the way of production of positrons and their subsequent annihilation with electrons, produce gamma photonselectromagnetic waves with even higher energy than X-rays.

Radiation dose depends on the exam performed where increased time that the X-ray beam is "on" results in a higher radiation dose to the patient and potentially to health-care workers with inadequate protection. ${ }^{3}$ Single shot X-ray exams, such as a chest X-ray, result in a small radiation dose since X-rays are produced for the study for $\sim 1$ second. $\mathrm{CT}$ exams require the $\mathrm{X}$-ray beam to be on for a longer time than a single shot film resulting in a higher radiation dose. Fluoroscopy, a study that requires multiple longer duration imaging runs, such as that used for a cerebral angiogram, results in very high radiation dose. This is especially true if an intervention, such as coil embolization of a cerebral aneurysm, is performed because an intervention often requires prolonged imaging compared with a simple diagnostic angiogram. This duration-verses-radiation dose effect is illustrated in - Table 1.

The ability of X-ray and gamma photons to ionize substances also contributes to their adverse effects. Ionization of molecular targets leads to breaking of covalent bonds and the formation of positively charged compounds, due to the loss of electrons, from substances that are normally electrically neutral. When these molecular targets are regulatory and structural proteins, enzymes, or nucleic acids, significant cellular dysfunction can result. In general, the cells most sensitive to radiation injury are those that are either rapidly dividing, such as bone marrow, or those that lead to the production of gametes-ovary and testis. However, one unique structure that is exquisitely sensitive to radiation injury is the lens of the eye. ${ }^{4}$ Although not due to cell killing, ocular radiation exposure can lead to cataractogenesis via a variety of mechanisms that include inducing excessive lens epithelial cell proliferation and altered differentiation as well
(C2021. Indian Society of Neuroanaesthesiology and Critical Care. This is an open access article published by Thieme under the terms of the Creative Commons Attribution-NonDerivative-NonCommercial-License, permitting copying and reproduction so long as the original work is given appropriate credit. Contents may not be used for commercial purposes, or adapted, remixed, transformed or built upon. (https://creativecommons.org/licenses/by-nc-nd/4.0/). Thieme Medical and Scientific Publishers Pvt. Ltd. A-12, 2nd Floor, Sector 2, Noida-201301 UP, India 
Table 1 Average patient radiation dose and increased fatal cancer risk associated with radiologic imaging

\begin{tabular}{|l|l|l|}
\hline Procedure & $\begin{array}{l}\text { Average } \\
\text { radiation } \\
\text { dose per test } \\
\text { (mSv) }\end{array}$ & $\begin{array}{l}\text { Lifetime increased } \\
\text { risk of fatal cancer } \\
\text { per test }\end{array}$ \\
\hline Chest X-ray & 0.1 & $1: 200,000$ \\
\hline CT of the head & 2 & $1: 10,000$ \\
\hline $\begin{array}{l}\text { Diagnostic cerebral } \\
\text { angiogram }\end{array}$ & 7 & $1: 3,000$ \\
\hline $\begin{array}{l}\text { Cerebral angiogram } \\
\text { with aneurysm coil } \\
\text { embolization }\end{array}$ & 15 & $1: 1,318$ \\
\hline
\end{tabular}

Abbreviations: $\mathrm{CT}$, computerized tomogram; mSv, millisievert. Note: Data are given as radiation dose per test expressed in $\mathrm{mSv}$ where, for X-ray imaging, $1 \mathrm{mSv}=1 \mathrm{~mJ}$ of energy absorbed per kilogram of tissue. $^{3}$ For reference, the average total radiation exposure per individual per year in the United States is $6.2 \mathrm{mSv} .{ }^{9}$ Data are also given for increased risk for fatal cancer due to radiation exposure. ${ }^{10}$

as post-transcriptional modifications to crystallin, a major structural protein of the lens. ${ }^{5}$ Therefore, for health-care providers who are not wearing lead-containing eyewear, standing behind a full-length lead shield offers greater protection than wearing a full lead apron and thyroid shield as the latter leaves the ocular lenses unprotected from the cataractogenic effects of X-ray exposure.

The "As Low As Reasonably Achievable," or ALARA principle, provides some guidance to decrease radiation exposure to both patients and health-care workers. ${ }^{6}$ First and foremost, one should determine if the test requiring radiation exposure is really necessary. If so, the exam should be performed with the goal of minimizing the duration that the $\mathrm{X}$-ray beam is "on" and using a device that provides a collimated beam. Most X-ray tubes do produce a collimated beam that is designed to restrict scattering of $\mathrm{X}$-rays beyond the structures to be imaged. Despite using a device with a beam collimator, X-ray protective lead blankets or aprons should be used to minimize residual radiation exposure to the patient's radiosensitive organs, if possible. Health-care worker should remain as far away from the X-ray tube as feasible and still employ radiation shielding devices such as lead aprons and lead thyroid shields, but, better yet, stand behind lead screens as the screen provides better ocular protection. One may even consider wearing lead-containing eyewear. Health-care workers who suspect to be or are pregnant should consider further minimizing or avoiding exposure to radiation, especially during the first trimester of their pregnancy. They should consider wearing a dosimeter near their abdomen but under their lead apron as lead only decreases but does not eliminate radiation exposure to supposed protected body parts. For example, lead aprons consisting of a layer of lead of 0.25 and $0.50 \mathrm{~mm}$ thickness will decrease transmitted radiation by 92.4 to $97.1 \%$ and 97.8 to $99.6 \%$, respectively, provided that the lead layer is completely intact and does not contain cracks that can occur with folding and handling of the apron. ${ }^{7,8}$

As anesthesiologists, we must be advocates for our patients and protect them from harm. Simultaneously, we must also protect ourselves. X-ray and gamma photons are invisible and are easily overlooked yet pose significant risk to humans. By the time my career as a neuroanesthesiologist comes to an end, I will have worked in the clinical setting for at least 30 years where I provide direct care to patients during countless CT and interventional radiological studiesthat's a lot of radiation exposure! We should all remember that, although radiation exposure to health-care workers is relatively low during a single test, cumulative exposure over the course of decades may be additive. Most health-care workers are generally good about wearing lead aprons and even thyroid shields-however, remember to protect your eyes. Awareness of the risk of radiation and ways to protect oneself from these risks now can really help to minimize risk for cancer and cataracts later.

\section{Conflict of Interest}

None declared.

\section{References}

1 United States Environmental Protection Agency. Radiation protection - radiation sources and doses. Available at: https:// www.epa.gov/radiation/radiation-sources-and-doses\#: :tex$\mathrm{t}=$ All\%20of\%20us\%20are\%20exposed,6.2\%20millisieverts\%20 (620\%20millirem). Accessed December 18, 2020

2 Berrington de Gonzalez A, Darby S. Risk of cancer from diagnostic X-rays: estimates for the UK and 14 other countries. Lancet 2004;363:345-351

3 Mettler FA Jr, Huda W, Yoshizumi TT, et al. Effective doses in radiology and diagnostic nuclear medicine: a catalog. Radiology 2008;248:254-263

4 International Commission on Radiological Protection. The 2007 Recommendations of the International Commission on Radiological Protection. ICRP Publication 103. Ann ICRP. 37(2-4). Available from: http://www.icrp.org/publication.asp?id=ICRP $\% 20$. Accessed December 28, 2020

5 Hamada N. Ionizing radiation sensitivity of the ocular lens and its dose rate dependence. Int J Radiat Biol 2017;93:1024-1034

6 Bevelacqua JJ. Practical and effective ALARA. Health Phys 2010; 98(Suppl 2):S39-S47

7 Simon SL. Organ-specific external dose coefficients and protective apron transmission factors for historical dose reconstruction for medical personnel. Health Phys 2011;101:13-27

8 Tan J, Brock-Utne JG. Did you know this about your lead apron? Anesth Analg 2013;117:534-535

9 National Council on Radiation Protection and Measurements. Report 160 - Ionizing radiation exposure of the population of the United States (2009). Available at: https://ncrponline.org/. Accessed December 28, 2020

10 American Society of Radiologist Technologists. Available at: www.xrayrisk.com. Accessed December 28, 2020 